University of Wollongong

Research Online

Faculty of Informatics - Papers (Archive)

Faculty of Engineering and Information

Sciences

2007

\title{
Mathematical modelling of the self-heating process in compost piles
}

Harvinder Sidhu

University of New South Wales

Mark Nelson

University of Wollongong, mnelson@uow.edu.au

Thiansiri Luangwilai

University of New South Wales

Xiao Dong Chen

Monash University

Follow this and additional works at: https://ro.uow.edu.au/infopapers

Part of the Physical Sciences and Mathematics Commons

\section{Recommended Citation}

Sidhu, Harvinder; Nelson, Mark; Luangwilai, Thiansiri; and Chen, Xiao Dong: Mathematical modelling of the self-heating process in compost piles 2007.

https://ro.uow.edu.au/infopapers/2671

Research Online is the open access institutional repository for the University of Wollongong. For further information contact the UOW Library: research-pubs@uow.edu.au 


\title{
Mathematical modelling of the self-heating process in compost piles
}

\begin{abstract}
We model the increase in temperature in compost piles or landfill sites due to micro-organisms undergoing exothermic reactions. The model incorporates the heat release due to biological activity within the pile and the heat release due to the oxidation of cellulosic materials. The heat release rate due to biological activity is modelled by a function which is a monotonic increasing function of temperature over a particular range and followed by a monotone decreasing function of temperature. This functionality represents the fact that micro-organisms die or become dormant at high temperatures. The heat release due to the oxidation reaction is modelled by the usual manner using Arrhenius kinetics. The bifurcation behaviour is investigated for two-dimensional slab geometries to determine the critical sizes of the compost piles.
\end{abstract}

Disciplines

Physical Sciences and Mathematics

\section{Publication Details}

Sidhu, H. S., Nelson, M. I., Luangwilai, T. \& Chen, X. (2007). Mathematical modelling of the self-heating process in compost piles. Chemical Product and Process Modeling, 2 (2), Article No. 8. 


\title{
Mathematical Modelling of the Self-Heating Process in Compost Piles
}

\author{
Harvinder S. Sidhu, Mark I. Nelson, Thiansiri Luangwilai, and Xiao Dong Chen
}

\begin{abstract}
We model the increase in temperature in compost piles or landfill sites due to microorganisms undergoing exothermic reactions. The model incorporates the heat release due to biological activity within the pile and the heat release due to the oxidation of cellulosic materials. The heat release rate due to biological activity is modelled by a function which is a monotonic increasing function of temperature over a particular range and followed by a monotone decreasing function of temperature. This functionality represents the fact that micro-organisms die or become dormant at high temperatures. The heat release due to the oxidation reaction is modelled by the usual manner using Arrhenius kinetics. The bifurcation behaviour is investigated for twodimensional slab geometries to determine the critical sizes of the compost piles.
\end{abstract}

KEYWORDS: self-heating, nonlinear dynamics, composting, combustion, safety 


\section{Introduction}

The phenomenon of spontaneous ignition due to internal heating in bulk solids such as coal, grain, hay, wool wastes, etc., can be described by thermal explosion theory (see Bowes, 1984, and Frank-Kamenetskii, 1969). In such models heat release is usually represented by a single Arrhenius reaction and combustion is initiated when heat-loss is unable to balance the heat generated by the internal heating of the bulk material. However in industrial processes involving large volumes of bulk organic materials there are in fact two sources of heat-generation: a low-temperature process involving the growth and respiration of micro-organisms, such as aerobic mould-fungi and bacteria, and a high-temperature process due to oxidation of cellulosic materials. Examples of processes where biological heating is important include large-scale composting operations (Rynk, 2000), the storage of industrial waste fuel, such as municipal solid waste, and landfills. In these examples self-heating due to biological activity is considered desirable, for example in composting (Brinton et al, 1995). Elevated temperatures of the order 70 to $90^{\circ} \mathrm{C}$ may be found within a few months or even a few days (Hogland et al., 1996). Although it has been recognised for over twenty years that "...biological heating may be an indispensable prelude to self-ignition..." (Bowes, 1984:373), very little information is available regarding the mechanism of fires when biological self-heating is involved. An understanding of this phenomenon is crucial as fires (most likely due to biological self-heating) are common at landfills worldwide (Hudak, 2001).

The case when self-heating is entirely due to cellulosic oxidation is well known (Bowes, 1984, and Frank-Kamenetskii, 1969). Nelson et al. (2003) analysed a spatially uniform model, based upon Semenovs theory for thermal explosions, for self-heating in compost piles. They investigated the cases when self-heating is due to purely biological heat-release and due to a combination of biological and chemical heat-release. Since the system investigated was described by a single (but nonlinear), first-order ordinary differential equations, with few parameters, the authors were able to thoroughly investigate the generic steady-state behaviour of the system when parameters were varied by using singularity theory (Golubitsky and Schaeffer, 1985). In this paper we consider a two-dimensional, spatially-dependent model that contains both biological and chemical activity. We believe that this preliminary analysis is a constructive step towards an understanding of the self-heating process in compost piles.

\section{Mathematical Formulation}

Ignoring the depletion of cellulosic materials, biomass and oxygen, the mathematical model of self-heating in a compost heap reduces to an energy equation. For 


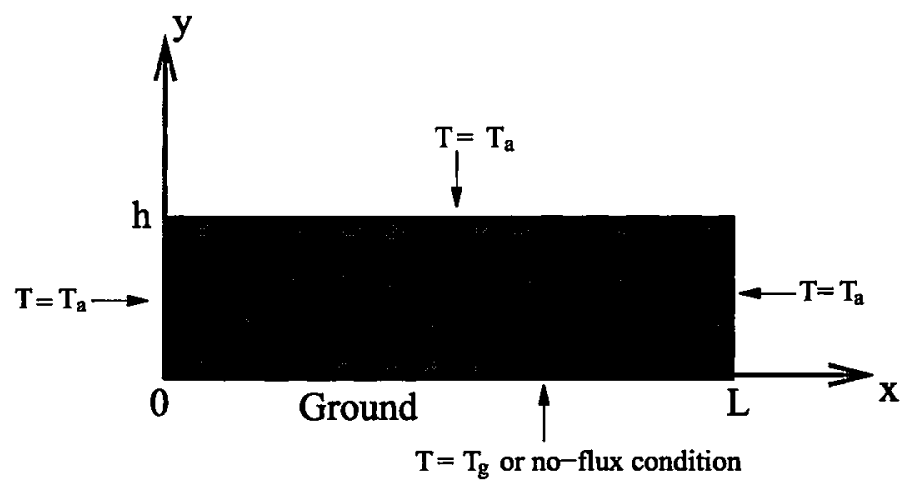

Figure 1: Schematic diagram showing the geometry of the two-dimensional compost pile with a rectangular cross-section and the corresponding boundary conditions.

simplicity, local thermal equilibrium is assumed which is a common assumption for porous medium and packed particle beds (Nield and Bejan, 1992). Thus interphase (solid particles and gas) temperature gradients are ignored and a single temperature is used to describe local behaviour. We will consider an idealized two-dimensional pile which has a domain described by a rectangular slab (width $L$ with a height $h$ ) shown in figure 1 . The figure also shows the boundary conditions that we have used in our numerical investigation of the model.

The relevant equations for the model are:

$$
\begin{gathered}
\text { Energy balance on } 0 \leq x \leq L, 0 \leq y \leq h \\
(\rho C)_{\mathrm{eff}} \frac{\partial T}{\partial t}=k_{\mathrm{eff}} \nabla^{2} T+Q_{c}(1-\varepsilon) \rho_{c} A_{c} \exp \left[\frac{-E_{c}}{R T}\right]+Q_{b}(1-\varepsilon) \rho_{b} \frac{A_{1} \exp \left[\frac{-E_{1}}{R T}\right]}{1+A_{2} \exp \left[\frac{-E_{2}}{R T}\right]}
\end{gathered}
$$

$$
\begin{gathered}
\text { Algebraic relationships } \\
k_{\mathrm{eff}}=\varepsilon k_{\mathrm{air}}+(1-\varepsilon) k_{\mathrm{c}} \\
(\rho C)_{\mathrm{eff}}=\varepsilon \rho_{\mathrm{air}} C_{\mathrm{air}}+(1-\varepsilon) \varepsilon \rho_{c} C_{c} .
\end{gathered}
$$

The terms that appear in equations (1) - (3) are defined in the nomenclature. The heat produced due to oxidation of the cellulosic material is represented by the second term on the right-hand side of the energy equation (1). However unlike previous investigations into spontaneous ignition in bulk solids, we have included the heat 
generated due to the biological activity within the pile - represented by the third term on the right-hand side of equation (1). In this term, the growth parameters $A_{1}$ and $E_{1}$ reflect that at low temperatures the metabolic activity of the biomass grows with increasing temperature as enzyme activity increases. However, for sufficiently high temperatures the essential proteins which are sensitive to heat begin to denature leading to cell death. These processes are represented by the biomass deactivation parameters $A_{2}$ and $E_{2}$. To ensure that the heat release due to the biological activity within the compost pile has a global maximum in the temperature range, the activation energy for the inhibition process must be larger than the activation energy for the biomass growth, i.e. $E_{2}>E_{1}$. The detailed formulation of the term representing the heat generated by the biomass can be found in Chen \& Mitchell (1996).

The algebraic expressions (2) and (3) give the relationships for the effective thermal conductivity and the effective thermal capacity of the compost pile in terms of corresponding properties of air and pure cellulosic material.

The formulation of this model is completed once the boundary conditions have been defined. The boundary conditions used in his study are specified in figure 1, and are given explicitly below:

$$
\begin{gathered}
\text { Boundary conditions } \\
T=T_{a} \quad \text { along } x=0, x=L \text { and } y=h, \\
T=T_{g} \text { or } \frac{\partial T}{\partial n}=0 \quad \text { along the base } y=0 . \\
\text { Initial Conditions } \\
T=T_{a},
\end{gathered}
$$

where $\partial T / \partial n$ describes the outward normal derivative at the boundary. We compare two cases representing the extremes when the base of the compost pile is a perfect insulator $(\partial T / \partial n=0)$, and when it is a perfect conductor $\left(T=T_{g}\right)$. Sexton et al. (2001) stated that the latter boundary condition appears to closely approximate the conditions currently found for bagasse piles, and note that the former condition has practical advantages but involves extra costs in implementation.

\section{Numerics}

In the next section we present results to the solutions of the governing equation (1) and the corresponding boundary conditions. These were obtained using a time and space adaptive finite element package FLEXPDE ${ }^{\mathrm{TM}}$ (PDE Solutions Inc). Due to its adaptive nature, errors in both space and time are minimized as mesh points are added in regions of large gradients particularly on the surface of the compost 
pile. All calculations are performed to a relative error tolerance of less than $0.1 \%$. Before the current two-dimensional investigation was undertaken, solutions obtained from FLEXPDE ${ }^{\mathrm{TM}}$ were verified by using Auto (Doedel et al., 1998) to solve the one-dimensional version of the governing equation. We note that Auto does not determine the stability of the steady-state solutions. However, by solving the timedependent version of the governing equation using FLEXPDE ${ }^{\mathrm{TM}}$, one can determine the stability of the solution branches. In all of our results, we shall only consider the stable solution branches.

\section{Results}

In this section we present the results from our numerical investigation of the model, equations (1) - (3). Since we are interested in the dimensions of the compost pile (in metres) and the corresponding temperature distribution within the pile (in Kelvins), we have decided to study the dimensional version of the governing equations. Our study is initially based on two different heights: $h=$ (total width of pile) $/ 4$, and $h=$ (total width of pile)/8. In other words, in sections 4.1 and 4.2, the ratios of the width to height of the rectangular pile are kept constant. Furthermore, we use two different boundary conditions at the base of the compost pile: an insulating (Neumann, or no-flux) condition, and a fixed temperature condition (Dirichlet, or perfectly conducting). In these sections we show that, for a given boundary condition and a given value of $h$, there are critical values of $L$, at which transitions between different types of self-heating behaviour occur. In section 4.3 we study how these critical values change as the height of the compost heap is varied.

The spatial profiles shown in figures 2 and 3 represent the cases when the base of the pile is perfectly insulated, and when the temperature of the base equal the ground temperature, respectively. In both of these cases the height of the pile is taken to be one-quarter of the total width of the pile.

Figure 2 shows that when the base of the pile is perfectly insulated, the maximum temperature of $372 \mathrm{~K}$ occurs at the point $(22,0)$, which is at the middle of the base of the pile, and the temperature decreases towards the value of the air temperature at the boundaries of the pile. Figure 3, on the other hand, shows that when the base of the pile is a perfect conductor $\left(T=T_{g}\right)$, the maximum temperature occurs at the middle of the compost pile. At this point, the maximum steady-state temperature was found to be $368.3 \mathrm{~K}$, and the temperature from the middle decreases to their corresponding values at the boundaries, i.e. ground temperature at the base, and air temperature at the other boundaries. 
Sidhu et al.: Modelling of Compost Piles

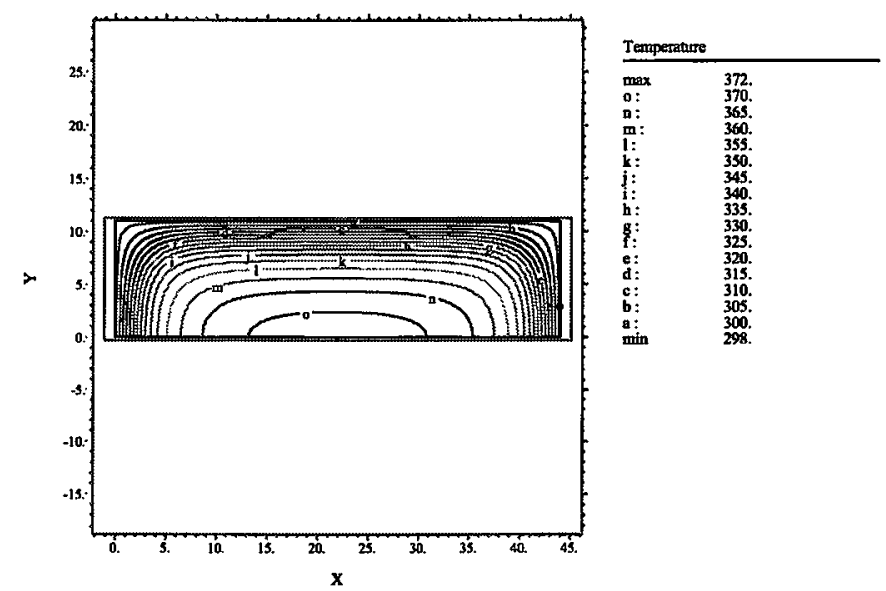

Figure 2: Steady-state temperature distribution within the compost pile for the no flux boundary condition at the base of the pile. The width of the compost pile is $44 \mathrm{~m}$, height is $11 \mathrm{~m}$ and the maximum temperature $T_{\max }=372 \mathrm{~K}$ occurs at the middle of the base of the pile, and the minimum temperature $T_{\min }=298 \mathrm{~K}$, occurs at the compost-air and -ground interfaces.

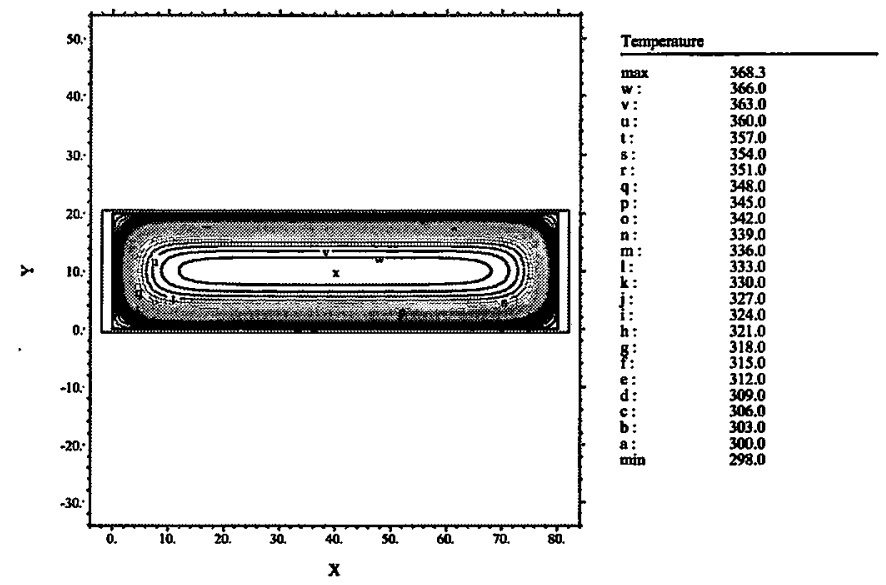

Figure 3: Steady-state temperature distribution within the compost pile when the boundary condition at the base of the pile is $T=T_{g}(=298 \mathrm{~K})$. The width of the compost pile is $80 \mathrm{~m}$, height is $20 \mathrm{~m}$ and the maximum temperature $T_{\max }=368.3 \mathrm{~K}$ occurs in the middle of the pile, and the minimum temperature $T_{\min }=298 \mathrm{~K}$, occurs at the compost-air and -ground interfaces. 
Chemical Product and Process Modeling, Vol. 2 [2007], Iss. 2, Art. 8

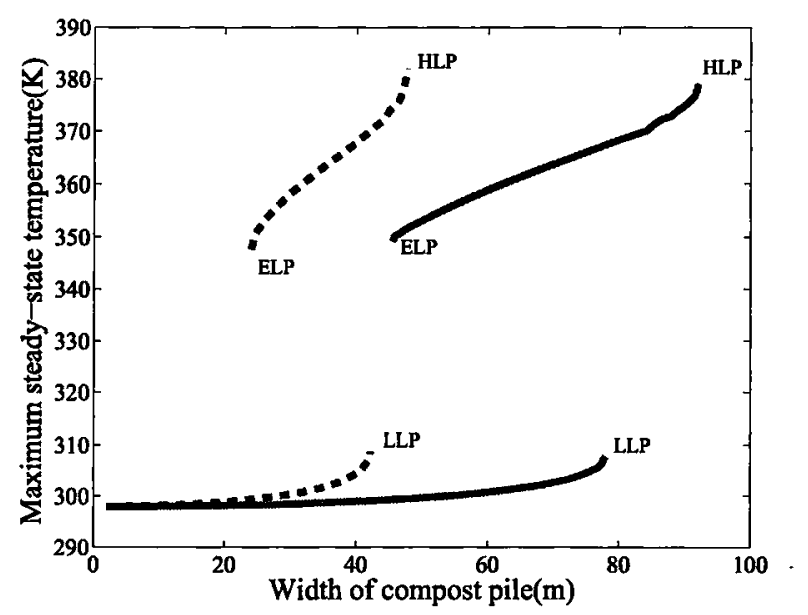

Figure 4: The dependence of maximum steady-state temperature within the compost heap upon the width of the pile when the height of the pile $=$ (total width)/4. The stable steady-state solution branch represented by the green bold curve corresponds to the boundary condition $T=T_{g}$ at the base, whereas the dashed blue curve uses the no-flux boundary condition at the base of the pile. The notation for the critical points is: LLP - low temperature ignition limit point; HLP - high temperature ignition limit point; ELP - extinction limit point.

\subsection{Results for $h=$ (total width) $/ 4$}

Figure 4 shows the case when the height of the pile is one quarter of the total width of the compost pile. We see that when the base of the compost heap is kept at ground temperature (bold green curve), the lowest solution branch, sometimes referred to as the no-reaction branch, ceases when $L=78 \mathrm{~m}$. We refer to this point as the low temperature ignition point (represented by the point LLP in the figure). If $L$ is taken slightly larger than this value, the numerical solution converges to the elevated solution branch. For example, if $L=79 \mathrm{~m}$ the maximum steady-state temperature is now $370 \mathrm{~K}$, as opposed to $307.7 \mathrm{~K}$ at the low temperature ignition point. This elevated stable solution branch exists until $L=92 \mathrm{~m}$, beyond which numerical solutions do not converge (the solution 'blows-up'). If $L$ is taken beyond this width (denoted by the point labeled HLP in the figure) the cellulosic materials ignite and flaming combustion occurs within the compost heap. We refer to this point as the high temperature ignition limit point. 
As we reduce the width from the high-temperature ignition limit, the elevated solution branch ceases at around $L=45.5 \mathrm{~m}$ (point ELP in figure 4). If we reduce the width of the heap below this critical value, the solution converges onto the low temperature, no-reaction branch. This point is often called the extinction limit point. For values of $L$ between the extinction point and the low-temperature ignition point, there are two low temperature stable solution branches: the no-reaction branch and the elevated temperature branch. From an operational viewpoint, the elevated temperature branch is desirable since the composting process is enhanced at such temperatures. Noting also that when temperatures reach beyond $80^{\circ} \mathrm{C}$, the microorganisms die, or become dormant and the biological activity ceases (Rynk, 2000).

When the insulated boundary condition is used at the base of the pile (dashed blue curve in figure 4), the general shape of the solution branch remains the same, i.e. the existence of the low temperature and high temperature ignition points, as well as the extinction point. However, the values of $L$ at which these critical points occur are lower for this case the low-temperature ignition limit point (LLP) occurs at $L=42.2 \mathrm{~m}$, the high temperature ignition limit point (HLP) occurs at $L=47.7 \mathrm{~m}$, and the extinction limit point (ELP) occurs for $L=24 \mathrm{~m}$. Hence the region of bistability, between the extinction and low temperature limit points, is narrower than for the case when the base temperature taken to equal the ground temperature.

\subsection{Results for $h=($ total width $) / 8$}

Figure 5 shows the results when the height of the compost heap is one-eighth the total width of the compost. In this case, the locations of all the critical points have increased (close to double the values found for the earlier case), and consequently the region of bi-stability has also increased. As in figure 4, the solutions corresponding to the insulated boundary condition at the base of the pile has lower $L$ values for the critical points than the solutions when the base temperature equals the ground temperature.

\subsection{Examining the critical points}

Figure 6 shows the locations of the three critical points, the low and high temperature ignition limit points, and the extinction limit point, for various ratios of height to total width of the compost heap. Here we only present the results for the insulated condition used at the base of the pile. Similar results to those shown in figure 6 were obtained for the case when the base temperature of the pile was fixed to equal the ground temperature. We note that the cases when the ratios were fixed at 0.125 and 0.25 have already been discussed in the previous two sections. 
Chemical Product and Process Modeling, Vol. 2 [2007], Iss. 2, Art. 8

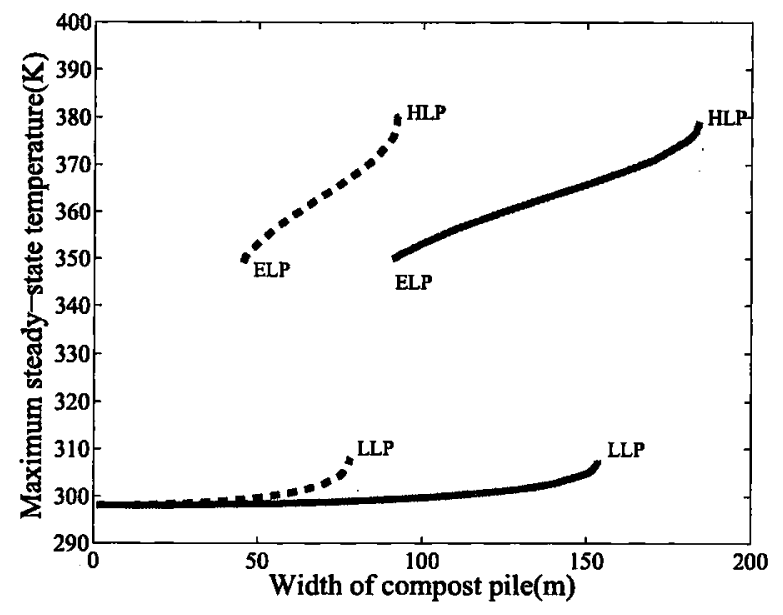

Figure 5: The dependence of maximum steady-state temperature within the compost heap upon the width of the pile when the height of the pile $=$ (total width)/8. The notation used here is the same as in figure 4 .

As expected, when the height of the pile is increased the locations of the critical values of $L$ reduce. Hence the region when the (desirable) elevated solution branch is possible narrows with increasing $h$. More importantly, when the heights are increased the high temperature ignition limit point (HLP) reduces to a value very close to the low temperature limit point (LLP), and when $h=0.75 \times$ (total width), these two limit points are almost the same (when these limit points are equal it is referred to as a double-limit point bifurcation). For such cases, any slight increase in the total width of the pile can result in the temperature of the cellulosic material moving from the low temperature, no-reaction branch, to the ignited (flaming combustion) state (since the solution does not converge onto the elevated solution branch as previously seen in figures 4 and 5). Similar behaviour was observed by Nelson et al. (2003) when the Semenov approach was used. This figure also implies that stacking new compost on top of an existing compost pile (increasing $h$ for fixed $L$ ) can cause a transition from an evaluated temperature to flaming combustion. This transition occurs because the elevated temperature branch that exists for the value of $L$ is now destroyed when $h$ is increased.

\section{Conclusions}

In this paper, we have undertaken a preliminary investigation of a two-dimensional, spatially dependent model for the self-heating of a compost heap. This model in- 


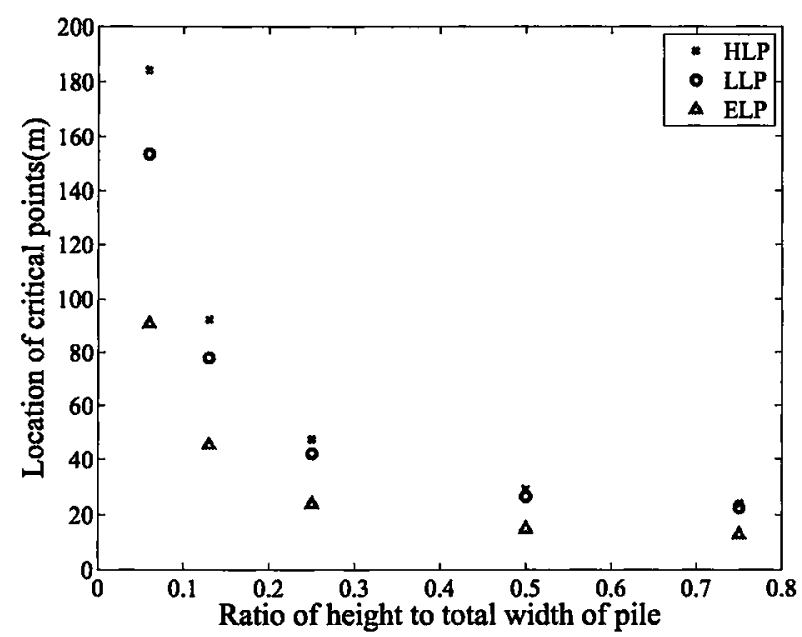

Figure 6: The locations of various critical points as functions of the height of the compost heap. The labels LLP, HLP and ELP refer to the low temperature ignition limit point, high temperature ignition limit point and the extinction limit point respectively.

corporates terms that account for both the biological and chemical activities which are known to occur within the compost heap. For simplicity we have assumed the geometry of the compost heap to be a two-dimensional rectangular slab.

The model exhibits two stable 'low temperature' branches. The lowest of these represents a state of negligible heat-reaction. This is undesirable. The higher of these is an elevated temperature branch, which is the feature of interest in industrial composting facilities and municipial tips. There is also a much higher temperature branch which represents flaming combustion within the compost heap. Transitions between these three types of behaviour are well-defined. Our results suggest that composting performance can be improved by insulating the lower boundary. Although highly simplified, the current model predicts the features of interest. Our results also seem to show that an insulated pile of height $h / 2$ gives similar results to a conducting pile of height $h$, as would be expected from symmetry conditions for similar ground and air temperatures.

We are currently working on extending the model to include oxygen consumption, and convection of oxygen into the pile. In addition to the industrial processes mentioned previously, it has long been suspected that the initiation of haystack fires is due to biological heating under the influence of water content or air humidity, which then increases the temperature sufficiently to trigger significant oxidation of 
cellulosic material (Bowes, 1984: Chapter 9). We hope to extend our model to include these features at a later date.

\section{NOMENCLATURE}

$A_{C} \quad$ Pre-exponential factor for the oxidation of the cellulosic material $\left(\mathrm{s}^{-1}\right)$

$A_{1} \quad$ Pre-exponential factor for the oxidation of the biomass growth $\left(\mathrm{s}^{-1}\right)$

$A_{2} \quad$ Pre-exponential factor for the inhibibition of biomass growth (-)

$C_{\text {air }} \quad$ Heat capacity of air $\left(\mathrm{J} \mathrm{kg}^{-1} \mathrm{~K}^{-1}\right)$

$C_{c} \quad$ Heat capacity of the cellulosic material $\left(\mathrm{J} \mathrm{kg}^{-1} \mathrm{~K}^{-1}\right)$

$E_{c} \quad$ Activation Energy for the oxidation of the cellulosic material $\left(\mathrm{J} \mathrm{mol}^{-1}\right)$

$E_{1} \quad$ Activation Energy for the biomass growth ( $\mathrm{J} \mathrm{mol} \mathrm{biomass}^{-1}$ )

$E_{2} \quad$ Activation energy for the inhibition of biomass growth (J mol biomass ${ }^{-1}$ )

$L \quad$ Width of the compost pile (m)

$Q_{b} \quad$ Exothermicity for the oxidation of biomass per $\mathrm{kg}$ of dry cellulose $\left(\mathrm{J} \mathrm{kg}^{-1}\right)$

$Q_{c} \quad$ Exothermicity for the oxidation of the cellulosic material $\left(\mathrm{J} \mathrm{kg}^{-1}\right)$

$R \quad$ Ideal gas constant $\left(\mathrm{J} \mathrm{K}^{-1} \mathrm{~mol}^{-1}\right)$

$T \quad$ Temperature within the compost pile (K)

$T_{a} \quad$ Ambient temperature (K)

$T_{g} \quad$ Temperature of the ground (K)

$h \quad$ Height of the compost pile (m)

$k_{\text {air }} \quad$ Effective thermal conductivity of air $\left(\mathrm{W} \mathrm{m}^{-1} \mathrm{~K}^{-1}\right)$

$k_{c} \quad$ Effective thermal conductivity of cellulose $\left(\mathrm{W} \mathrm{m}^{-1} \mathrm{~K}^{-1}\right)$

$k_{\text {eff }} \quad$ Effective thermal conductivity of the bed $\left(\mathrm{W} \mathrm{m}^{-1} \mathrm{~K}^{-1}\right)$

$t \quad$ Time (s)

$x \quad$ Spatial distance along the width of the pile $(\mathrm{m})$

$y \quad$ Spatial distance along the height of the pile (m)

$\varepsilon \quad$ Void fraction (-)

$(\rho C)_{\text {eff }}$ Effective thermal capacity per unit volume of the bed $\left(\mathrm{J} \mathrm{m}^{-3} \mathrm{~K}^{-1}\right)$

$\rho_{\text {air }} \quad$ Density of air $\left(\mathrm{kg} \mathrm{m}^{-3}\right)$

$\rho_{b} \quad$ Density of bulk biomass within the compost pile $\left(\mathrm{kg} \mathrm{m}^{-3}\right)$

$\rho_{c} \quad$ Density of pure cellulosic material $\left(\mathrm{kg} \mathrm{m}^{-3}\right)$

Unless otherwise specified we take the following typical values:

$$
\begin{aligned}
& A_{C}=1.8 \times 10^{4} \mathrm{~s}^{-1}, A_{1}=2.0 \times 10^{6} \mathrm{~s}^{-1}, A_{2}=6.86 \times 10^{30}, C_{\text {air }}=1005 \mathrm{~J} \mathrm{~kg}^{-1} \mathrm{~K}^{-1}, \\
& C_{c}=3320 \mathrm{~J} \mathrm{~kg}^{-1} \mathrm{~K}^{-1}, E_{c}=1.1 \times 10^{5} \mathrm{~J} \mathrm{~mol}^{-1}, E_{1}=1.0 \times 10^{5} \mathrm{~J} \mathrm{~mol} \mathrm{biomass}^{-1}, \\
& \left.E_{2}=2.0 \times 10^{5} \mathrm{~J} \mathrm{~mol} \mathrm{biomass}^{-1}\right), Q_{b}=6.66 \times 10^{6} \mathrm{~J} \mathrm{~kg}^{-1}, Q_{c}=1.7 \times 10^{7} \mathrm{~J} \mathrm{~kg}^{-1}, \\
& R=8.314 \mathrm{~J} \mathrm{~K}^{-1} \mathrm{~mol}^{-1}, T_{a}=298 \mathrm{~K}, T_{g}=298 \mathrm{~K}, k_{\text {air }}=0.026 \mathrm{~W} \mathrm{~m}^{-1} \mathrm{~K}^{-1}, k_{c}=0.3
\end{aligned}
$$


Sidhu et al.: Modelling of Compost Piles

$\left.\mathrm{W} \mathrm{m} \mathrm{m}^{-1} \mathrm{~K}^{-1}, \varepsilon=0.3, \rho_{\text {air }}=1.17 \mathrm{~kg} \mathrm{~m}^{-3}, \rho_{b}=575 \mathrm{~kg} \mathrm{~m}^{-3}, \rho_{c}=1150 \mathrm{~kg} \mathrm{~m}^{-3}\right)$.

\section{REFERENCES}

Bowes, P.C., "Self-heating: evaluating and controlling the hazard", Elsevier, Amsterdam (1984).

Brinton, W.F., Evans, Jr.E., Droffner, M.L. \& Brinton, R.B., "Standartized test for evaluation of compost self-heating", BioCycle, vol. 36, 60-65 (1995).

Chen, X.D. \& Mitchell, D.A., "Start-up strategise for self-heating and efficient growth in stirred bioreactors for solid state fermentation", Proceedings of the 24th Annual Australian and New Zealand Chemical Engineering Conference, 4, The Institute of Engineers, 111-116 (1996).

Doedel, E.J., Fairgrieve, T.F., Sandstede, B., Champneys, A.R., Kuznetsov, Y.A. and Wang, X., "AUTO97: Continuation and bifurcation software for Ordinary Differential Equations (with Hom-Cont)". Available by anonymous ftp from

ftp.cs. concordia.ca/pub/doedel/auto (1998).

FLEXPDE ${ }^{\mathrm{TM}}$, PDE Solutions, Inc., http://www.pdesolutions . com

Frank-Kamenetskii, D.A., "Diffusion and heat transfer in chemical kinetics", New York: Plenum Press (Second Edition) (1969).

Golubitsky, M. \& Schaeffer, D., "The classification theorem in Singularities and Groups in Bifurcation Theory", 1st edition, Springer, Berlin Germany (1985).

Hogland, W., Bramryd, T. \& Persson, 1., "Physical, biological and chemical effects of unsorted fractions of industrial solid waste in waste fuel storage", Waste Management \& Research, vol. 14, 197-210 (1996).

Hudak, P.F., "Spontaneous combustion of shale spoils at sanitary landfill", Waste Management \& Research, vol. 22, 687-688 (2001).

Nelson, M.I., Balakrishnan, E. \& Chen, X.D., "A Semenov model of self-heating in compost piles", Transactions of IChemE Part B, vol. 81, 375-383 (2003).

Nield, D. and Bejan, A., "Convection in porous media", Springer-Verlag, New York (1992). 
Chemical Product and Process Modeling, Vol. 2 [2007], Iss. 2, Art. 8

Rynk, R., "Fires at composting facilities", BioCycle Magazine, vol. 41, 54-58 (2000).

Sexton, M.J., Macaskill, C. \& Gray, B.F., "Self-heating and drying in two-dimensional bagasse piles", Combustion Theory and Modelling, vol. 5, 517-536 (2001). 